PROCEEDINGS OF THE

AMERICAN MATHEMATICAL SOCIETY

Volume 139, Number 8, August 2011, Pages 2729-2733

S 0002-9939(2011)10955-9

Article electronically published on March 24, 2011

\title{
ON EXTREMAL QUASICONFORMAL MAPPINGS OF NON-LANDSLIDE TYPE
}

\author{
FAN JINHUA
}

(Communicated by Mario Bonk)

\begin{abstract}
Let $S=\{(x, y): 0<x<1,0<y<1\}$, and let $f$ be a quasiconformal mapping on $S$. It is proved that there is at least one extremal quasiconformal mapping of non-landslide type in the Teichmüller equivalence class $[f]$. This gives a positive answer to the problem proposed by $\mathrm{Z}$. Li in a recent paper.
\end{abstract}

\section{INTRODUCTION}

Let $\Delta$ be the open unit disk in the complex plane. For a quasiconformal mapping $f$ on $\Delta$, we let $\mu_{f}=\frac{f_{\bar{z}}}{f_{z}}$ be the Beltrami coefficient of $f$ and let $K(f)=\frac{1+\left\|\mu_{f}\right\|_{\infty}}{1-\left\|\mu_{f}\right\|_{\infty}}$ be the maximal dilatation of $f$. A quasiconformal mapping $f$ on $\Delta$ induces a homomorphism $\left.f\right|_{\partial \Delta}$ on $\partial \Delta$. Two quasiconformal mappings $f$ and $g$ on $\Delta$ are Teichmüller equivalent if $\left.f\right|_{\partial \Delta}=\left.g\right|_{\partial \Delta}$, and we let $[f]$ be the Teichmüller equivalence class of $f$.

A quasiconformal mapping $f$ on $\Delta$ is extremal when $K(f) \leq K(g)$ for any $g \in[f]$. If $[f]$ contains infinitely many extremal quasiconformal mappings, then there is an extremal quasiconformal mapping $g \in[f]$ such that the set

$$
E_{g}(\delta)=\left\{z \in \Delta: \frac{1+\left|\mu_{g}(z)\right|}{1-\left|\mu_{g}(z)\right|}<K(f)-\delta\right\}
$$

has a positive measure for some $\delta>0$ (refer to [6], [11 for the proof). For the details of extremal quasiconformal mappings theory, we refer to [1, [2], [5], 7], 8], [9].

When the set $E_{f}(\delta)$ has interior points for some $\delta>0$, we say the extremal quasiconformal mapping $f$ is of landslide type. Otherwise, an extremal quasiconformal mapping $f$ is of non-landslide type when the set $E_{f}(\delta)$ has no interior points for any $\delta>0$. So it is natural to ask the following question (refer to [4, [11]).

Problem 1. Suppose that $f$ is a quasiconformal mapping on $\Delta$ and $[f]$ contains infinitely many extremal quasiconformal mappings. Is it true that $[f]$ always contains an extremal quasiconformal mapping of landslide type?

In 4, Li proved the following theorem.

Received by the editors July 1, 2010.

2010 Mathematics Subject Classification. Primary 30C75.

Key words and phrases. Extremal quasiconformal mapping, variability set, non-landslide type. The author was supported by Tian-Yuan Foundation (No. 10926159) and NJUST (XKF09044).

(C)2011 American Mathematical Society Reverts to public domain 28 years from publication 
Theorem 1.1 (4]). There exists a quasiconformal mapping $f$ on $\Delta$ such that there are infinitely many extremal quasiconformal mappings of non-landslide type in $[f]$.

The motivation of Theorem 1.1 was to give a negative answer to Problem 1. However, we still do not know the answer to Problem 1. In 4, Li proposed the following problem.

Problem 2. For any given quasiconformal mappings $f$ of $\Delta$, is there always an extremal quasiconformal mapping $g \in[f]$ of non-landslide type?

The purpose of this paper is to give Problem 2 a positive answer. For convenience of statement, we choose $S=\{(x, y): 0<x<1,0<y<1\}$ as our consideration and prove the following theorem.

Theorem 1.2. Let $f$ be a quasiconformal mapping on $S$. Then there exists at least one extremal quasiconformal mapping $g \in[f]$ of non-landslide type.

Furthermore, using the methods in the proof of Theorem 1.2, as a generalization of Theorem 1.1, we get the following theorem.

Theorem 1.3. Let $f$ be a quasiconformal mapping on $S$. If $[f]$ contains infinitely many extremal quasiconformal mappings, then there always exist infinitely many extremal quasiconformal mappings of non-landslide type in $[f]$.

\section{Proof of theorems}

In this section, by some lemmas, we will prove Theorems 1.2 and 1.3.

Lemma 2.1 ([3]). Let $\left\{f_{n}\right\}$ be a sequence of $K$-quasiconformal mappings of $\Omega$ in the complex plane which converges locally to a quasiconformal mapping $f$ with Beltrami coefficient $\mu$. If the Beltrami coefficients $\mu_{n}$ of $f_{n}$ tend to a limit a.e., then $\lim _{n \longrightarrow \infty} \mu_{n}(z)=\mu(z)$ a.e.

Lemma 2.2 ([10]). Let $f$ be a quasiconformal mapping of the unit disk $\Delta$ onto itself. Choose a fixed point $z_{0} \in \Delta$ and, for $w \in \Delta$, let $g$ be an extremal quasiconformal mapping $\Delta$ onto itself which is equal to $f$ on $\partial \Delta$ and takes $z_{0}$ into $w$. Let $K$ be the maximal dilatation of $g$. Then $K$ is, for fixed $z_{0}$, a continuous function of $w$ which tends to infinity for $|w| \longrightarrow 1$.

Let $f$ be a quasiconformal mapping on $\Delta$. We call

$$
V\left[z_{0}\right]=\left\{w=g\left(z_{0}\right), g \text { is extremal in }[f]\right\}
$$

the variability set of $z_{0}$.

Lemma 2.3 (10]). Let $f$ be an extremal quasiconformal mapping of the unit disk $\Delta$ onto itself with maximal dilatation $K_{0}$, and let $w$ be a point outside the variability set $V\left[z_{0}\right]$. Let $g$ be an extremal quasiconformal mapping of $\Delta$ onto itself with the boundary values $\left.f\right|_{\partial \triangle}$ and $g\left(z_{0}\right)=w$. Then $\mu_{g}=k \frac{\bar{\varphi}}{|\varphi|}$, where $\frac{1+k}{1-k}>K_{0}$ and $\varphi$ is analytic in $\Delta \backslash z_{0}$.

Using Lemmas 2.2 and 2.3, we have the following lemma.

Lemma 2.4. Let $f$ be a quasiconformal mapping of the unit disk $\Delta$ onto itself with maximal dilatation $K_{0}$. Then, for any $K>K_{0}$, there exists a quasiconformal $g \in[f]$ such that $\left|\mu_{g}(z)\right|=k, z \in \Delta$, where $k=\frac{K-1}{K+1}$. 
Proof. Let $\widetilde{f} \in[f]$ be an extremal quasiconformal mapping. Then $K(\widetilde{f}) \leq K_{0}$. For $z_{0} \in \Delta$ and $K>K_{0} \geq K(\widetilde{f})$, by Lemma 2.2 , we can choose a point $w \notin V\left[z_{0}\right]$ and $g \in[f]$ such that $K(g)=K$ and $g\left(z_{0}\right)=w$. For $w \notin V\left[z_{0}\right]$, by Lemma 2.3, we have $\left|\mu_{g}(z)\right|=k, z \in \Delta$.

Proof of Theorem 1.2. Let $S=\{(x, y): 0<x<1,0<y<1\}$, and let $f$ be a quasiconformal mapping on $S$. We will construct an extremal quasiconformal mapping $g \in[f]$ of non-landslide type. With no loss of generality, we suppose $f$ is extremal and set $K=K(f), k=\frac{K-1}{K+1}$. If $[f]$ contains a uniquely extremal quasiconformal mapping $f$, then the theorem holds for $f$ of non-landslide type. So we suppose that $[f]$ contains infinitely many extremal quasiconformal mappings. We will prove the theorem by the following four steps.

Step 1. A sequence of open squares $\left\{S_{j}^{(n)}\right\}$ and a sequence of extremal quasiconformal mappings $\left\{f^{(n)} \in[f]\right\}$ are constructed.

$n=1$. Dividing $S$ by the lines $x=\frac{1}{2}$ and $y=\frac{1}{2}$, we get $4^{1}$ open squares with side length $\frac{1}{2}$ denoted by

$$
S_{1}^{(1)}, S_{2}^{(1)}, S_{3}^{(1)}, S_{4}^{(1)} .
$$

For each $S_{j}^{(1)}(j=1,2,3,4)$, we define a quasiconformal mapping $f_{j}^{(1)}$ on $S_{j}^{(1)}$ as follows. If $\exists \delta>0$ such that $K\left(\left.f\right|_{S_{j}^{(1)}}\right)<K-\delta$, applying Lemma 2.4 for $\left.f\right|_{S_{j}^{(1)}}$, we can define a quasiconformal mapping $f_{j}^{(1)}$ on $S_{j}^{(1)}$ such that $\left.f_{j}^{(1)}\right|_{\partial S_{j}^{(1)}}=\left.f\right|_{\partial S_{j}^{(1)}}$ and $\left|\mu_{f_{j}^{(1)}}(z)\right|=k$ for all $z \in S_{j}^{(1)}$. Otherwise, there is no $\delta>0$ such that $K\left(\left.f\right|_{S_{j}^{(1)}}\right)<$ $K-\delta$, and we define the quasiconformal mapping $f_{j}^{(1)}=\left.f\right|_{S_{j}^{(1)}}$.

Then we define $f^{(1)}$ on $S$ as

$$
f^{(1)}(z)=f_{j}^{(1)}(z), \quad z \in \overline{S_{j}^{(1)}},(j=1,2,3,4) .
$$

By the definition of $f^{(1)}$, we have that $f^{(1)} \in[f]$ is extremal and $K\left(\left.f^{(1)}\right|_{S_{j}^{(1)}}\right)=$ $K\left(f_{j}^{(1)}\right)=K$.

$n-1 \longrightarrow n$. For each square $S_{j}^{(n-1)}\left(j=1,2, \cdots, 4^{n-1}\right)$, as was done to $S$, we divide $S_{j}^{(n-1)}$ into four congruent squares. Then we get $4^{n}$ open squares with side length $\frac{1}{2^{n}}$ denoted by

$$
S_{1}^{(n)}, S_{2}^{(n)}, \cdots, S_{j}^{(n)}, \cdots, S_{4^{n}}^{(n)} .
$$

Similar to the definition of $f_{j}^{(1)}$ on $S_{j}^{(1)}$, we define $f_{j}^{(n)}$ on $S_{j}^{(n)}$ as follows. If $\exists \delta>0$ such that $K\left(\left.f^{(n-1)}\right|_{S_{j}^{(n)}}\right)<K-\delta$, applying Lemma 2.4 for $\left.f^{(n-1)}\right|_{S_{j}^{(n)}}$, we can define a quasiconformal mapping $f_{j}^{(n)}$ on $S_{j}^{(n)}$ such that $\left.f_{j}^{(n)}\right|_{\partial S_{j}^{(n)}}=\left.f^{(n-1)}\right|_{\partial S_{j}^{(n)}}$ and $\left|\mu_{f_{j}^{(n)}}(z)\right|=k$ for all $z \in S_{j}^{(n)}$. Otherwise, there is no $\delta>0$ such that $K\left(\left.f^{(n-1)}\right|_{S_{j}^{(n)}}\right)<K-\delta$, and we define the quasiconformal mapping $f_{j}^{(n)}=$ $\left.f^{(n-1)}\right|_{S_{j}^{(n)}}$. Then we define $f^{(n)}$ on $S$ as

$$
f^{(n)}(z)=f_{j}^{(n)}(z), \quad z \in \overline{S_{j}^{(n)}},\left(j=1,2, \cdots, 4^{n}\right) .
$$


By the definition of $f^{(n)}$, we have that $f^{(n)} \in[f]$ is extremal and $K\left(\left.f^{(n)}\right|_{S_{j}^{(n)}}\right)=$ $K\left(f_{j}^{(n)}\right)=K,\left(j=1,2, \cdots, 4^{n}\right)$.

So we have constructed a sequence of open squares $\left\{S_{j}^{(n)}, j=1,2, \cdots, 4^{n}\right\}$ and a sequence of extremal quasiconformal mappings $\left\{f^{(n)} \in[f]\right\}$ such that

$$
K\left(\left.f^{(n)}\right|_{S_{j}^{(n)}}\right)=K\left(f_{j}^{(n)}\right)=K .
$$

Step 2. For the normality of quasiconformal mappings, there is a subsequence of $\left\{f^{(n)}\right\}$ (we denote it also by $\left\{f^{(n)}\right\}$ ) that converges locally to a quasiconformal mapping $g$. It is obvious that $g \in[f]$ is extremal.

Step 3. Let $\mu$ be the Beltrami coefficient of $g$ and let $\mu_{n}$ be the Beltrami coefficient of $f^{(n)}$. We claim that $\lim _{n \longrightarrow \infty} \mu_{n}(z)=\mu(z)$ a.e. $z \in S$. By Lemma 2.1, it is sufficient to prove that $\mu_{n}$ a.e. tends to a limit. When the two-dimensional measure of $\bigcup_{n=1}^{\infty} \bigcup_{j=1}^{4^{n}} \partial S_{j}^{n}$ is 0 , let $z \in S \backslash \bigcup_{n=1}^{\infty} \bigcup_{j=1}^{4^{n}} \partial S_{j}^{n}$. We will prove that $\mu_{n}(z)$ tends to a limit.

By the definition of $\left\{f^{(n)}\right\}$, we have the following properties of $\mu_{n}$. If $K\left(\left.f^{(n)}\right|_{S_{j_{n+1}}^{(n+1)}}\right)=K$ for all $n$ and squares $S_{j_{n+1}}^{(n+1)} \ni z$, then $\left.f^{(n+1)}\right|_{S_{j_{n+1}}^{(n+1)}}=\left.f\right|_{S_{j_{n+1}}^{(n+1)}}$, so $\lim _{n \longrightarrow \infty} \mu_{n}(z)=\mu_{f}(z)$. Otherwise, if there exists $\delta>0, m$ and $S_{j_{m+1}}^{(m+1)} \ni z$ such that $K\left(\left.f^{(m)}\right|_{S_{j_{m+1}}^{(m+1)}}\right)<K-\delta$, then $\left.f^{(n)}\right|_{S_{j_{n}}^{(n)}}=\left.f^{(m+1)}\right|_{S_{j_{n}}^{(n)}}$ for all $n \geq m+1$ and $S_{j_{n}}^{(n)} \ni z$, so $\lim _{n \longrightarrow \infty} \mu_{n}(z)=\mu_{f^{(m+1)}}(z)$.

Step 4. We claim that $g \in[f]$ obtained in Step 2 is of non-landslide type. If, on the contrary, $g$ is of landslide type, then there is a $\delta>0$ and an open subset $E \in S$ such that $K\left(\left.g\right|_{E}\right)<K-\delta$. For $E$ an open set, we fix $m$ and $j_{m}$ such that $S_{j_{m}}^{(m)} \subset E$. Then

$$
K\left(\left.g\right|_{S_{j m}^{(m)}}\right) \leq K\left(\left.g\right|_{E}\right)<K-\delta .
$$

If $K\left(\left.f^{(n-1)}\right|_{S_{j_{n}}^{(n)}}\right)=K$ for all $n>m$ and $S_{j_{n}}^{(n)} \subset S_{j_{m}}^{(m)}$, by the argument in Step 3, we have $\left.f^{(n)}\right|_{S_{j_{n}}^{(n)}}=\left.f^{(m)}\right|_{S_{j_{n}}^{(n)}}$ for all $n>m$, so

$$
\left.\mu\right|_{S_{j_{m}}^{(m)}}=\left.\lim _{n \longrightarrow \infty} \mu_{n}\right|_{S_{j_{m}}^{(m)}}=\left.\mu_{f^{(m)}}\right|_{S_{j_{m}}^{(m)}} .
$$

It follows from (2.1) and (2.3) that

$$
K\left(\left.g\right|_{S_{j_{m}}^{(m)}}\right)=K
$$

Otherwise, if there exists $\delta>0, n$ and $S_{j_{n}}^{(n)} \subset S_{j_{m}}^{(m)}$ such that $K\left(\left.f^{(n-1)}\right|_{S_{j_{n}}^{(n)}}\right)<$ $K-\delta$, we have $\left.f^{(l)}\right|_{S_{j_{n}}^{(n)}}=\left.f^{(n)}\right|_{S_{j_{n}}^{(n)}}$ for all $l \geq n$, so

$$
\left.\mu\right|_{S_{j_{n}}^{(n)}}=\left.\lim _{l \longrightarrow \infty} \mu_{l}\right|_{S_{j_{n}}^{(n)}}=\left.\mu_{f(n)}\right|_{S_{j_{n}}^{(n)}} .
$$

It follows from (2.1) and (2.5) that

$$
K\left(\left.g\right|_{S_{j_{m}}^{(m)}}\right) \geq K\left(\left.g\right|_{S_{j_{n}}^{(n)}}\right)=K .
$$

However, (2.2) contradicts (2.4) and (2.6), so $g \in[f]$ is an extremal quasiconformal mapping of non-landslide type. This completes the proof. 
Proof of Theorem 1.3. Let $K(f)=K$ and $k=\frac{K-1}{K+1}$. If any extremal quasiconformal mapping in $[f]$ is of non-landslide type, the theorem holds when $[f]$ contains infinitely many extremal quasiconformal mappings. If there is an extremal quasiconformal mapping $g \in[f]$ of landslide type, this means that there is a $\delta>0$ and an open subset $E \in S$ such that $K\left(\left.g\right|_{E}\right)<K-\delta$. Let $z_{0} \in E, w_{0}=g\left(z_{0}\right)$ and $L_{\theta}=\left\{w, \arg \left(w-w_{0}\right)=\theta\right\}(\theta \in(-\pi, \pi])$. Applying Lemma 2.4 for $g \mid E$, we get $w_{\theta} \in L_{\theta}$ and a quasiconformal mapping $g_{\theta} \in[f]$ such that

$$
\left.g_{\theta}\right|_{\partial E}=\left.g\right|_{\partial E},\left|\mu_{g_{\theta}}(z)\right|=k \text { for all } z \in E \text {, and } g_{\theta}\left(z_{0}\right)=w_{\theta} .
$$

Starting with the extremal quasiconformal mapping $g_{\theta}$, following the proof of Theorem 1.2, we can construct an extremal quasiconformal mapping $\widetilde{g}_{\theta} \in[f]$ of non-landslide type. By the construction of $\widetilde{g}_{\theta}$ and $\left|\mu_{g_{\theta}}(z)\right|=k$ for all $z \in E$, we have $\left.\widetilde{g}_{\theta}\right|_{E}=\left.g_{\theta}\right|_{E}$. Furthermore, by (2.7), if $\theta_{1} \neq \theta_{2}$,

$$
\widetilde{g}_{\theta_{1}}\left(z_{0}\right)=g_{\theta_{1}}\left(z_{0}\right)=w_{\theta_{1}} \neq w_{\theta_{2}}=g_{\theta_{2}}\left(z_{0}\right)=\widetilde{g}_{\theta_{2}}\left(z_{0}\right)
$$

so $\widetilde{g}_{\theta_{1}} \neq \widetilde{g}_{\theta_{2}}$. Varying $\theta \in(-\pi, \pi]$, we get infinitely many extremal quasiconformal mappings $\widetilde{g}_{\theta} \in[f]$ of non-landslide type, which completes the proof.

\section{ACKNOWLEDGMENT}

The author would like to thank the anonymous referee for suggestions which improved the quality of this paper.

\section{REFERENCES}

1. V. Bozin, N. Lakic, V. Markovic, M. Mateljevic, Unique extremality, J. Anal. Math. 75 (1998), 299-337. MR1655836 (2000a:30045)

2. R. S. Hamilton, Extremal quasiconformal mappings with prescribed boundary values, Trans. Amer. Math. Soc. 138 (1969), 399-406. MR0245787 (39:7093)

3. O. Lehto, Univalent functions and Teichmüller spaces, GTM 109, Springer-Verlag, New York, 1987. MR 867407 (88f:30073)

4. Z. Li, A note on extremal quasi-conformal mappings, Sci. China Ser. A 53 (2010), 63-70. MR.2594747

5. S. L. Krushkal, Extremal quasiconformal mappings, Siberian Math. J. 10 (1969), 411-418.

6. E. Reich, Non-uniquely extremal quasiconformal mappings, Libertas Math. 20 (2000), 33-38. MR.1801108 (2002a:30033)

7. E. Reich, The unique extremality counterexample, J. Anal. Math. 75 (1998), 339-347. MR 1655837 (99k:30037)

8. E. Reich, Extremal quasiconformal mappings of the disk, in Handbook of Complex Analysis: Geometry Function Theory, vol. 1, North-Holland, 2002, pp. 75-136. MR1966190 (2004c:30036)

9. E. Reich, K. Strebel, Extremal quasiconformal mappings with given boundary values, in Contribution to Analysis, Academic Press, New York, 1974, pp. 375-392. MR0361065 (50:13511)

10. K. Strebel, Point shift differentials and extremal quasiconformal mappings, Ann. Acad. Sci. Fenn. Math. 23 (1998), 475-494. MR1642146 (99j:30022)

11. Z. Zhou, J. Chen, Z. Yang, On the extremal sets of extremal quasiconformal mappings, Sci. China Ser. A 46 (2003), 552-561. MR2014487(2004j:30036)

Department of Applied Mathematics, Nanjing University of Science and Technology, Nanjing, 210094, People's Republic of China

E-mail address: jinhuafan@hotmail.com 